\title{
Properly Coloured Cycles and Paths: Results and Open Problems
}

\author{
Gregory Gutin* $\quad$ Eun Jung $\mathrm{Kim}^{\dagger}$
}

\begin{abstract}
In this paper, we consider a number of results and six conjectures on properly coloured (PC) paths and cycles in edge-coloured multigraphs. We overview some known results and prove new ones. In particular, we consider a family of transformations of an edge-coloured multigraph $G$ into an ordinary graph that allow us to check the existence of PC cycles and PC $(s, t)$-paths in $G$ and, if they exist, to find shortest ones among them. We raise a problem of finding the optimal transformation and consider a possible solution to the problem.
\end{abstract}

\section{Introduction}

The class of edge-coloured multigraphs generalize directed graphs. There are several other generalizations of directed graphs such as arc-coloured digraphs, hypertournaments and star hypergraphs, but the class of edgecoloured multigraphs has been given the main attention in graph theory literature because many concepts and results on directed graphs can be extended to edge-coloured multigraphs and there are several important applications of edge-coloured multigraphs. For instance, in [10,11] Dorninger considers chromosome arrangement in a cell of an eukaryotic organism by using the 2-edge-coloured multigraphs. For a more extensive treatment of this topic, see $[6,7]$.

In this paper we overview some known results on properly coloured (PC) cycles and paths in edge-coloured multigraphs, prove new ones and consider several open problems on the topic. In Section 2 we briefly consider a problem of whether an edge-coloured graph has a PC cycle. In Sections 3 and 4, we offer a useful tool to study edge-coloured multigraphs. In investigating problems on PC subgraphs of edge-coloured multigraphs, it is convenient

\footnotetext{
*Department of Computer Science, Royal Holloway, University of London, Egham, Surrey TW20 0EX, UK, gutin@cs.rhul.ac.uk

${ }^{\dagger}$ Department of Computer Science, Royal Holloway, University of London, Egham, Surrey TW20 0EX, UK, eunjung@cs.rhul.ac.uk
} 
to transform an edge-coloured graph into an ordinary graph. We suggest a new technique that somewhat automates this transformation. Moreover, by proving some new results, we illustrate how the proposed technique allows us to obtain more efficient algorithms for PC cycle and PC $(s, t)$-path problems by reducing the order and size of the transformed graph. We raise a problem of determining the minimum order and size of the transformed graph, and describe the family of graphs that may be the solution to the problem.

In Section 5 we study long PC cycles and paths in arbitrary edge-coloured multigraphs and Section 6 is devoted to longest (mostly Hamilton) PC cycles in edge-coloured complete graphs.

An $m$-path-cycle subgraph $F$ of a multigraph $G$ is a vertex-disjoint union of $m$ paths and a number of cycles in $G$ (some cycles can be of length 2 ). If $m=0$, we call $F$ a cycle subgraph of $G$. For a vertex set $X$ of a multigraph $G, G\langle X\rangle$ denotes the subgraph of $G$ induced by $X$. For a pair $s, t$ of distinct vertices of $G$, a path between $s$ and $t$ is called an $(s, t)$-path.

We consider edge-coloured multigraphs, i.e., undirected multigraphs in which each edge has a colour, but no parallel edges have the same colour. If an edge-coloured multigraph $G$ has $c$ colours, we assume that the colours are $1,2, \ldots, c$ and we call $G$ a $c$-edge-coloured multigraph. We denote the colour of an edge $e$ of an edge-coloured multigraph $G$ by $\chi(e)$. When $G$ has no parallel edges, we call $G$ an edge-coloured graph.

Let $G$ be a $c$-edge-coloured multigraph and let $v \in V(G)$. By $N_{i}(v)$ we denote the set of neighbours of $v$ adjacent to $v$ by an edge of colour $i$; let $d_{i}(x)=\left|N_{i}(x)\right|$. The maximum (minimum) monochromatic degree of $G=(V, E)$ is defined by

$$
\begin{aligned}
& \Delta_{\text {mon }}(G)=\max \left\{d_{j}(v): v \in V, 1 \leq j \leq c\right\} \\
& \left(\delta_{\text {mon }}(G)=\min \left\{d_{j}(v): v \in V, 1 \leq j \leq c\right\}\right) .
\end{aligned}
$$

Let $\chi(v)=\left\{i: 1 \leq i \leq c, N_{i}(v) \neq \emptyset\right\}$. A path or cycle $Q$ of $G$ is properly coloured (PC) if every two adjacent edges of $Q$ are of different colours.

\section{Existence of PC Cycles}

Since a pair of parallel edges in a $c$-edge-coloured multigraph $(c \geq 2)$ forms a PC cycle, in this section, we consider only $c$-edge-coloured graphs.

It is easy to see that the problem of checking whether a $c$-edge-coloured graph has a PC cycle is more general (even for $c=2$ ) than the simple problem of verifying whether a digraph contains a directed cycle. Indeed, consider a digraph $D$ and, to obtain a 2-edge-coloured graph $G$ from $D$, replace each arc $x y$ of $D$ with edges $x z_{x y}$ and $z_{x y} y$ of colours 1 and 2, where 
$z_{x y}$ is a new vertex $\left(z_{x y} \neq z_{x^{\prime} y^{\prime}}\right.$ provided $\left.x y \neq x^{\prime} y^{\prime}\right)$. Observe that $G$ has a PC cycle if and only if $D$ has a directed cycle.

The following theorem by Yeo [21] provides a simple recursive way of checking whether a $c$-edge-coloured graph has a PC cycle. (For $c=2$, Theorem 2.1 was first proved by Grossman and Häggkvist [14].)

Theorem 2.1. Let $G$ be a c-edge-coloured graph, $c \geq 2$, with no $P C$ cycle. Then, $G$ has a vertex $z \in V(G)$ such that no connected component of $G-z$ is joined to $z$ with edges of more than one colour.

Let us consider the following function introduced by Gutin [15]: $d(n, c)$, the minimum number $k$ such that every $c$-edge-coloured graph of order $n$ and minimum monochromatic degree at least $k$ has a $\mathrm{PC}$ cycle. It was proved in [15] that $d(n, c)$ exists and that

$$
d(n, c) \leq \frac{1}{\lfloor c / 2\rfloor}\left(\log _{2} n-\frac{1}{3} \log _{2} \log _{2} n+\Theta(1)\right) .
$$

Abouelaoualim et al. [1] stated a conjecture which implies that $d(n, c)=1$ for each $c \geq 2$. Using a recursive construction inspired by Theorem 2.1 of $c$-edge-coloured graphs with minimum monochromatic degree $p$ and without PC cycles, Gutin [15] showed that

$$
d(n, c) \geq \frac{1}{c}\left(\log _{c} n-\log _{c} \log _{c} n\right)
$$

and, thus, the conjecture does not hold. The bounds (1) and (2) imply that $d(n, c)=\Theta\left(\log _{2} n\right)$ for every fixed $c \geq 2$.

Conjecture 2.2. [15] There is a function $s(c)$ dependent only on $c$ such that $d(n, c)=s(c) \log _{2} n(1+o(1))$.

In particular, it would be interesting to determine $s(2)$.

\section{P-Gadgets}

We consider gadget constructions which generalize some known constructions mentioned below. The P-gadget graphs $G^{*}$ and $G^{* *}$ of an edge-coloured multigraph $G$ described in the next section allow one to transform several problems on properly coloured subgraphs of $G$ into perfect matching problems in $G^{*}$ or $G^{* *}$.

Let $G$ be an edge-coloured multigraph and let $G^{\prime}=G-\{x \in V(G)$ : $|\chi(x)|=1\}$. For each $x \in V\left(G^{\prime}\right)$ let $G_{x}$ be an arbitrary (non-edge-coloured) graph with the following four properties:

$\mathbf{P 1}\left\{x_{q}: q \in \chi(x)\right\} \subseteq V\left(G_{x}\right)$ 
P2 $G_{x}$ has a perfect matching;

P3 For each $p \neq q \in \chi(x)$, if the graph $G_{x}-\left\{x_{p}, x_{q}\right\}$ is not empty, it has a perfect matching;

P4 For each set $L \subseteq \chi(x)$ with at least 3 elements; if the graph $G_{x}-\left\{x_{l}\right.$ : $l \in L\}$ is not empty, it has no perfect matching.

Each $G_{x}$ with the properties P1-P4 is called a $\mathbf{P}$-gadget. Let us consider the following three P-gadgets; the first two are known in the literature and the third one is new.

1. One P-gadget is due to Szeider [19]:

$$
\begin{gathered}
V\left(G_{x}\right)=\left\{x_{i}, x_{i}^{\prime}: i \in \chi(x)\right\} \cup\left\{x_{a}^{\prime \prime}, x_{b}^{\prime \prime}\right\} \text { and } \\
E\left(G_{x}\right)=\left\{x_{i}^{\prime} x_{a}^{\prime \prime}, x_{i}^{\prime} x_{b}^{\prime \prime}, x_{i} x_{i}^{\prime}: i \in \chi(x)\right\} \cup\left\{x_{a}^{\prime \prime} x_{b}^{\prime \prime}\right\} .
\end{gathered}
$$

We will call this the SP-gadget.

2. Another gadget is due to Bang-Jensen and Gutin [4]:

$$
V\left(G_{x}\right)=\left\{x_{j}: j \in \chi(x)\right\} \cup\left\{y_{j}: j \in \chi(x) \backslash\{m, M\}\right\},
$$

where $m=\min \chi(x), M=\max \chi(x)$, and

$E\left(G_{x}\right)=\left\{x_{j} y_{k}: j \in \chi(x), k \in \chi(x) \backslash\{m, M\}\right\} \cup\left\{x_{j} x_{k}: j \neq k \in \chi(x)\right\}$.

We will call this the BJGP-gadget.

3. The following new gadget is a sort of crossover of the above two and is called the $\mathbf{X P}$-gadget:

$$
V\left(G_{x}\right)=\left\{x_{j}: j \in \chi(x)\right\} \cup\left\{y_{j}: j \in \chi(x) \backslash\{m, M\}\right\},
$$

where $m$ and $M$ are defined above, and

$$
E\left(G_{x}\right)=\left\{x_{m} x_{M}\right\} \cup\left\{x_{j} y_{j}, x_{m} y_{j}, x_{M} y_{j}: j \in \chi(x) \backslash\{m, M\}\right\} .
$$

It is not difficult to verify that the tree P-gadgets indeed satisfy P1-P4. Let $z=\chi(x)$. Observe that the SP-gadget has $2 z+2$ vertices and $3 z+1$ edges, the BJGP-gadget $2 z-2$ vertices and $z(3 z-5) / 2$ edges, the XP-gadget $2 z-2$ vertices and $3 z-5$ edges. Thus, the XP-gadget has the minimum number of vertices and edges among the three P-gadgets. It is not difficult to verify that the XP-gadget has the minimum number of vertices and edges among all possible P-gadgets for $z=2,3,4$. Perhaps, this is true for any $z$.

Conjecture 3.1. The XP-gadget has the minimum number of vertices and edges among all possible P-gadgets for every $z \geq 2$.

We will see in the next section why minimizing the numbers of vertices and edges in P-gadgets is important for speeding up some algorithms on edge-coloured multigraphs. 


\section{$4 \quad$ P-gadget Graphs}

Let $G$ be a $c$-edge-coloured multigraph and let $G_{x}$ be a P-gadget for $x \in$ $V\left(G^{\prime}\right)$. The graph $G^{*}$ is defined as follows: $V\left(G^{*}\right)=\cup_{x \in V\left(G^{\prime}\right)} V\left(G_{x}\right)$ and $E\left(G^{*}\right)=E_{1} \cup E_{2}$, where $E_{1}=\cup_{x \in V\left(G^{\prime}\right)} E\left(G_{x}\right)$ and $E_{2}=\left\{y_{q} z_{q}: y, z \in\right.$ $\left.V\left(G^{\prime}\right), y z \in E(G), \chi(y z)=q, 1 \leq q \leq c\right\}$.

Let $s, t$ be a pair of distinct vertices of $G$ and let $H=G-\{s, t\}$. Let $G^{* *}$ be constructed from $H^{*}$ by adding $s$ and $t$ and edges $E_{3}=\left\{s x_{i}: s x \in\right.$ $E(G), \chi(s x)=i\} \cup\left\{t x_{i}: t x \in E(G), \chi(t x)=i\right\}$.

We will denote the number of vertices and edges in multigraphs $G, G^{*}$ and $G^{* *}$ by $n, m, n^{*}, m^{*}, n^{* *}$ and $m^{* *}$, respectively.

The following result relates perfect matchings of $G^{*}$ with PC cycle subgraphs of $G$. PC cycle subgraphs are important in several problems on edgecoloured multigraphs (for example, for the PC Hamilton cycle problem described in Section 6), see [6]. Recall that $G^{\prime}=G-\{x \in V(G):|\chi(x)|=1\}$.

Theorem 4.1. Let $G$ be a connected edge-coloured multigraph such that $G^{\prime}$ is non-empty. Then $G$ has a PC cycle subgraph with $r$ edges if and only if $G^{*}$ has a perfect matching with exactly $r$ edges in $E_{2}$.

Proof: Let $M$ be a perfect matching of $G^{*}$ with exactly edges

$$
x_{p_{1}}^{1} y_{q_{1}}^{1}, \ldots, x_{p_{r}}^{r} y_{q_{r}}^{r}
$$

in $E_{2}$. For a vertex $x$ of $G^{\prime}$, let $Q_{x}$ be the set of edges in $E_{2}$ adjacent to $G_{x}$. By P2, each $G_{x}$ has even number of vertices $\left(x \in V\left(G^{\prime}\right)\right)$ and since $M$ is a perfect matching in $G^{*}$, there is even number of edges in $Q_{x}$. By $\mathrm{P} 4, Q_{x}$ has either no edges or two edges for each $x \in V\left(G^{\prime}\right)$. Let $X$ be the set of all vertices $x \in V\left(G^{\prime}\right)$ such that $\left|Q_{x}\right|=2$. Then, by the definition of $G^{*}, G\langle X\rangle$ contains a $\mathrm{PC}$ cycle factor. It remains to observe that $|X|=r$.

Now let $F$ be a PC cycle subgraph of $G$ with $r$ edges. Observe that the edges of $F$ correspond to a set $Q$ of $r$ independent edges of $G^{*}$ and that either no edges or two edges of $Q$ are adjacent to $G_{x}$ for each $x \in V\left(G^{\prime}\right)$. Now delete the vertices adjacent with $Q$ from each $G_{x}$ and observe that each remaining non-empty gadget has a perfect matching by P2 and P3. Combining the perfect matchings of the non-empty gadgets with $Q$, we get a perfect matching of $G^{*}$ with exactly $r$ edges from $E_{2}$.

The first part of the next assertion generalizes a result from [4]. The second part is based on an approach which leads to a more efficient algorithm than in $[2]$.

Corollary 4.2. One can check whether an edge-coloured multigraph $G$ has a $P C$ cycle and, if it does, find a maximum $P C$ cycle subgraph of $G$ in time $O\left(n^{*} \cdot\left(m^{*}+n^{*} \log n^{*}\right)\right)$. Moreover one can find a shortest PC cycle in $G$ in time $O\left(n \cdot n^{*} \cdot\left(m^{*}+n^{*} \log n^{*}\right)\right)$. 
Proof: We may assume that $G$ is connected and that $G^{\prime}$ is not empty. By Theorem 4.1, it is enough to find a perfect matching of $G^{*}$ containing the maximum number of edges from $E_{2}$. Assign weight 0 (1, respectively) to edges of $G^{*}$ in $E_{1}\left(E_{2}\right.$, respectively). Now we need to find a maximum weight perfect matching of $G^{*}$ which can be done in time $O\left(n^{*} \cdot\left(m^{*}+n^{*} \log n^{*}\right)\right)$ by a matching algorithm in [13].

To find a shortest PC cycle in $G$, choose a vertex $x \in V\left(G^{\prime}\right)$. We will find a shortest PC cycle in $G$ traversing $x$. By Theorem 4.1, it is enough to find a perfect matching of $G^{*}$ containing the minimum number of edges from $E_{2}$ while containing at least one edge from $E_{2}$ so that the corresponding PC cycle in $G$ should be non-trivial. We define the weights on edges of $G^{*}$ as follows. Assign $M$, where $M$ is a sufficiently large number, to each edge in $E_{2}$ incident with $G_{x}$. For all other edges, assign weight 1 (0, respectively) to edges of $G^{*}$ in $E_{1}\left(E_{2}\right.$, respectively). A maximum weight perfect matching of $G^{*}$ contains exactly two edges of weight $M$ by P4, and contains the minimum number of edges in $E_{2}$. Finding a maximum weight perfect matching of $G^{*}$ can be done in time $O\left(n^{*} \cdot\left(m^{*}+n^{*} \log n^{*}\right)\right)$ and we iterate the process for each $x \in V\left(G^{\prime}\right)$.

The proof of the following result is analogous to the proof of Theorem 4.1 .

Theorem 4.3. Let $G$ be an edge-coloured multigraph and let $s, t$ be a pair of distinct vertices of $G$. If $G^{* *}$ is non-empty, then $G$ has a PC 1-path-cycle subgraph with $r$ edges in which the path is between $s$ and $t$ if and only if $G^{* *}$ has a perfect matching with exactly $r$ edges not in $E_{1}$.

The next assertion generalizes a result from [2].

Corollary 4.4. Let $G$ be an edge-coloured multigraph. One can check whether there is a PC $(s, t)$-path in $G$ in time $O\left(m^{* *}\right)$ and if $G$ has one, a shortest $P C(s, t)$-path can be found in time $O\left(n^{* *} \cdot\left(m^{* *}+n^{* *} \log n^{* *}\right)\right)$.

Proof: Let $L$ be a graph. Given a matching $M$ in $L$, a path $P$ in $L$ is $M$-augmenting if, for any pair of adjacent edges in $P$, exactly one of them belongs to $M$ and the first and last edges of $P$ do not belong to $M$. Consider a perfect matching $M$ of $H^{*}$, where $H=G-\{s, t\}$, which is a collection of perfect matchings of $G_{x}$ for all $x \in V\left(G^{\prime}\right)$. The existence of a perfect matching in $G_{x}$ is guaranteed by $\mathrm{P} 2$. Observe that $G$ has a $\mathrm{PC}$ $(s, t)$-path if and only if there is an $M$-augmenting $(s, t)$-path $P$ in $G^{* *}$. Since an $M$-augmenting path $P$ can be found in time $O\left(m^{* *}\right)$ (see [20]), we can find a $\mathrm{PC}(s, t)$-path in $G$, if one exists, in time $O\left(m^{* *}\right)$.

To find a shortest PC $(s, t)$-path, we assign each edge in $\bigcup_{x \in V\left(G^{\prime}\right)} E\left(G_{x}\right)$ weight 0 and every other edge of $G^{* *}$ weight 1 . Observe that a minimum 
weight perfect matching $Q$ in the weighted $G^{* *}$ corresponds to a shortest $\mathrm{PC}(s, t)$-path. Finding a minimum weight perfect matching can be done in time $O\left(n^{* *} \cdot\left(m^{* *}+n^{* *} \log n^{* *}\right)\right)$.

\section{$5 \quad$ Long PC Cycles and Paths}

The following interesting result and conjecture were obtained by Abouelaoualim, Das, Fernandez de la Vega, Karpinski, Manoussakis, Martinhon and Saad [1].

Theorem 5.1. [1] Let $G$ be a c-edge-coloured multigraph $G$ with $n$ vertices and with $\delta_{\text {mon }}(G) \geq\left\lceil\frac{n+1}{2}\right\rceil$. If $c \geq 3$ or $c=2$ and $n$ is even, then $G$ has a Hamilton $P C$ cycle. If $c=2$ and $n$ is odd, then $G$ has a $P C$ cycle of length $n-1$.

Conjecture 5.2. [1] Theorem 5.1 holds if we replace $\delta_{\text {mon }}(G) \geq\left\lceil\frac{n+1}{2}\right\rceil$ by $\delta_{\text {mon }}(G) \geq\left\lceil\frac{n}{2}\right\rceil$.

We cannot replace $\delta_{\text {mon }}(G) \geq\left\lceil\frac{n+1}{2}\right\rceil$ by $\delta_{\text {mon }}(G) \geq\left\lceil\frac{n-1}{2}\right\rceil$ due to the following example. Let $H_{1}$ and $H_{2}$ be $c$-edge-coloured complete multigraphs (for each pair $x, y$ of vertices and each $i \in\{1,2, \ldots, c\}$ and $j \in\{1,2\}, H_{j}$ has a edge between $x$ and $y$ of colour $i$ ) of order $p+1$ that have precisely one vertex in common. Clearly, a longest $\mathrm{PC}$ cycle in $H_{1} \cup H_{2}$ is of length $p+1$.

Since the longest PC path problem is $\mathcal{N} \mathcal{P}$-hard, it makes sense to study lower bounds on the length of a longest PC path. The following result was proved by Abouelaoualim et al. [1].

Theorem 5.3. Let $G$ be a c-edge-coloured graph of order $n$ with $\delta_{\text {mon }}(G)=$ $d \geq 1$. Then $G$ has a $P C$ path of length at least $\min \left\{n-1,2\left\lfloor\frac{c}{2}\right\rfloor d\right\}$.

The authors of [1] raised the following two conjectures.

Conjecture 5.4. Let $G$ be a c-edge-coloured graph of order $n$ and let $d=$ $\delta_{\text {mon }}(G) \geq 1$. Then $G$ has a $P C$ path of length at least $\min \{n-1,2 c d\}$.

They also conjectured the following analog of Theorem 5.3 for multigraphs:

Conjecture 5.5. Let $G$ be a c-edge-coloured multigraph of order $n$ with $\delta_{\text {mon }}(G)=d \geq 1$. Then $G$ has a PC path of length at least $\min \{n-1,2 d\}$.

\section{Longest PC Cycles and Paths in Edge-Coloured Complete Graphs}

Let $K_{n}^{c}$ denote a $c$-edge-coloured complete graph with $n$ vertices.

Feng, Giesen, Guo, Gutin, Jensen and Rafiey [12] proved the following: 
Theorem 6.1. A $K_{n}^{c}(c \geq 2)$ has a PC Hamilton path if and only if $K_{n}^{c}$ contains a PC spanning 1-path-cycle subgraph.

This theorem was first proved by Bang-Jensen and Gutin [4] for the case $c=2$ and they conjectured that Theorem 6.1 holds for each $c \geq 2$. Theorem 6.1 implies that the maximum order of a PC path in $K_{n}^{c}$ equals the maximum order of a PC 1-path-cycle subgraph of $K_{n}^{c}$.

As a result, the problem of finding a longest $\mathrm{PC}$ path in $K_{n}^{c}$ is polynomialtime solvable for arbitrary $c \geq 2$. To see that a PC 1-path-cycle subgraph of $K_{n}^{c}$ can be found in polynomial time, add a pair $x, y$ of new vertices to $K_{n}^{c}$ together with all edges needed to have a complete graph on $n+2$ vertices. Let the colour of all edges between $x$ and $y$, and $K_{n}^{c}$ be $c+1$ and let the colour of $x y$ be $c+2$. Observe that the maximum order of a PC 1-path-cycle subgraph of $K_{n}^{c}$ is less by exactly two than the maximum order of a PC cycle subgraph of the $c+2$-edge-coloured complete graph described above. It remains to apply Corollary 4.2 .

The problem of finding a longest PC cycle $K_{n}^{c}$ has not been solved yet for $c \geq 3$ as we will see below. For $c=2$, Saad [17] found a characterization for longest PC cycles using the following notions. A pair of distinct vertices $x, y$ of $G$ are colour-connected if there exist PC $(x, y)$-paths $P$ and $Q$ such that $\chi\left(f_{P}\right) \neq \chi\left(f_{Q}\right)$ and $\chi\left(\ell_{P}\right) \neq \chi\left(\ell_{Q}\right)$, where $f_{P}$ and $f_{Q}$ are the first edges of $P$ and $Q$, respectively, and $\ell_{P}$ and $\ell_{Q}$ are the last edges of $P$ and $Q$, respectively. We say that $G$ is colour-connected if every pair of distinct vertices of $G$ is colour-connected. Saad's characterization is as follows.

Theorem 6.2. The length of a longest $P C$ cycle in a colour-connected $K_{n}^{2}$ is equal to the maximum order of a PC cycle subgraph of $K_{n}^{2}$.

Colour-connectivity for $K_{n}^{c}$ is an an equivalence relation (see [6]). Using Theorem 6.2, Saad [17] showed that the problem of finding a longest PC cycle in $K_{n}^{2}$ has a polynomial-time randomized algorithm. Using a special case of Corollary 4.2, Bang-Jensen and Gutin [5] proved that the problem is, in fact, polynomial-time solvable. Theorem 6.2 implies the following:

Corollary 6.3. [17] A $K_{n}^{2}$ has a PC Hamilton cycle if and only if $K_{n}^{2}$ is colour-connected and contains a PC cycle factor.

There is another characterization of $K_{n}^{2}$ with a PC Hamilton cycle due to Bankfalvi and Bankfalvi, see [6]. The straightforward extension of Corollary 6.3 is not true for any $c \geq 3$ [6]. In fact, no characterization of $K_{n}^{c}$ with a PC Hamilton cycle is known for any fixed $c \geq 3$ and it is a very interesting problem to obtain such a characterization. Even the following problem by Benkouar, Manoussakis, Paschos and Saad [8] is still open.

Problem 6.4. Determine the complexity of the PC Hamilton cycle problem for c-edge-coloured complete graphs when $c \geq 3$. 
We conjecture that the PC Hamilton cycle problem for $c$-edge-coloured complete graphs when $c \geq 3$ is polynomial-time solvable.

In absence of characterization of $K_{n}^{c}$ with a PC Hamilton cycle, sufficient conditions are interest. Manoussakis, Spyratos, Tuza and Voigt [16] proved the next result.

Proposition 6.5. If $c \geq \frac{1}{2}(n-1)(n-2)+2$, then every $K_{n}^{c}$ has a $P C$ Hamilton cycle.

Let $\Delta_{m o n}\left(K_{n}^{c}\right)$ denote the largest monochromatic degree of $K_{n}^{c}$. Bollobás and Erdös [9] posed the following:

Conjecture 6.6. Every $K_{n}^{c}$ with $\Delta_{\text {mon }}\left(K_{n}^{c}\right) \leq\lfloor n / 2\rfloor-1$ has a PC Hamilton cycle.

Improving some previous results on this conjecture, Shearer [18] showed that if $7 \Delta_{m o n}\left(K_{n}^{c}\right)<n$, then $K_{n}^{c}$ has a PC Hamilton cycle. So far, the best asymptotic estimate was obtained by Alon and Gutin [3].

Theorem 6.7. [3] For every $\epsilon>0$ there exists an $n_{0}=n_{0}(\epsilon)$ so that for each $n>n_{0}$, every $K_{n}^{c}$ satisfying $\Delta_{\text {mon }}\left(K_{n}^{c}\right) \leq\left(1-\frac{1}{\sqrt{2}}-\epsilon\right) n$ contains a $P C$ Hamilton cycle.

\section{References}

[1] A. Abouelaoualim, K.Ch. Das, W. Fernandez de la Vega, Y. Manoussakis, C.A. Martinhon, and R. Saad, Cycles and paths in edge-colored graphs with given degrees, Manuscript, 2007.

[2] A. Abouelaoualim, K.Ch. Das, L. Faria, Y. Manoussakis, C.A. Martinhon, and R. Saad, Paths and Trails in Edge-Colored Graphs, Proc. LATIN'08, Lecture Notes Comput. Sci., 4957 (2008), 723-735.

[3] N. Alon and G. Gutin, Properly colored Hamilton cycles in edge colored complete graphs. Random Struct. \& Alg., 11 (1997), 179-186.

[4] J. Bang-Jensen and G. Gutin, Alternating cycles and paths in edge-coloured multigraphs: a survey, Discrete Math., 165-166 (1997), 39-60.

[5] J. Bang-Jensen and G. Gutin, Alternating cycles and trails in 2-edge-coloured multigraphs, Discrete Math., 188 (1998), 61-72.

[6] J. Bang-Jensen and G. Gutin, Digraphs: Theory, Algorithms and Applications, Springer, London, 2000, Freely available online at www.cs.rhul.ac.uk/books/dbook/.

[7] J. Bang-Jensen and G. Gutin, Digraphs: Theory, Algorithms and Applications, 2nd Edition, Springer, London, In preparation.

[8] A. Benkouar, Y. Manoussakis, V. Paschos and R. Saad, On the Complexity of Some Hamiltonian and Eulerian Problems in Edge-colored Complete Graphs, Lect. Notes Computer Sci., 557 (1991), 190-198. 
[9] B. Bollobás and P. Erdős, Alternating Hamiltonian cycles, Israel J. Math., 23 (1976), 126-131.

[10] D. Dorninger, On permultations of chromesomes, Contributions to General Algebra, 5 (1987), Teubner-Verlag, Stuttgart, 95-103.

[11] D. Dorninger, Hamiltonian circuits determining the order of chromosomes, Discrete Appl. Math., 50 (1994), 159-168.

[12] J. Feng, H.-E. Giesen, Y. Guo, G. Gutin, T. Jensen and A. Rafiey, Characterization of edge-colored complete graph s with properly colored Hamilton paths, J. Graph Theory, 53 (2006), 333-346.

[13] H.N. Gabow, Data structures for weighted matching and nearest common ancestors with linking, Proc. SODA'90, 1990, 434-443.

[14] J.W. Grossman and R. Häggkvist, Alternating cycles in edge-partitioned graphs, J. Combin. Theory Ser. B 34 (1983), 77-81.

[15] G. Gutin, Note on edge-colored graphs and digraphs without properly colored cycles. Austral. J. Combin., To appear.

[16] Y. Manoussakis, M. Spyratos, Zs. Tuza and M. Voigt, Minimal colorings for properly colored subgraphs, Graphs \& Combin., 12 (1996), 345-360.

[17] R. Saad, Finding a longest alternating cycle in a 2-edge-coloured complete graph is in RP, Combin. Prob. \& Comput., 5 (1996), 297-306.

[18] J. Shearer, A property of the colored complete graph, Discrete Math., 25 (1979), 175-178.

[19] S. Szeider, Finding paths in graphs avoiding forbidden transitions, Discrete Appl. Math., 126 (2003), 261-273.

[20] R.E. Tarjan, Data structures and network algorithms, SIAM-Philadelphia, 1983.

[21] A. Yeo, A note on alternating cycles in edge-coloured graphs, J. Combin. Theory Ser. B 69 (1997), 222-225. 